\title{
The Use of Antimicrobial Photodynamic Therapy to Maintain a Hopeless Tooth With a Periodontic- Endodontic Lesion: A Case Report
}

\author{
Nahid Derikvand ${ }^{1}$, Masoud Hatami2 ${ }^{*}$, Nasim Chiniforush ${ }^{3}$, Seyedeh Sara Ghasemi ${ }^{4}$ \\ ${ }^{1}$ Department of Periodontics, Faculty of Dentistry, Borujerd Branch, Islamic Azad University, Borujerd, Iran \\ ${ }^{2}$ Department of Oral and Maxillofacial Medicine, School of Dentistry, Kermanshah University of Medical Sciences, \\ Kermanshah, Iran \\ ${ }^{3}$ Dental Implant Research Center, Dentistry Research Institute, Tehran University of Medical Sciences, Tehran, Iran \\ ${ }^{4}$ Dentist, Private Practice, Khorramabad, Iran
}

\author{
*Correspondence to \\ Masoud Hatami, DDS, MSC, \\ Department of Oral and \\ Maxillofacial Medicine, School \\ of Dentistry, Kermanshah \\ University of Medical Sciences, \\ Kermanshah, Iran. Tel: +98- \\ 9121962762 \\ +98-83327291448; \\ Email: \\ Masoud.Hatami@kums.ac.ir
}

Published online June 21, 2020

\begin{abstract}
Background: In spite of some advances in periodontal generative methods, it is impossible to stop progressive Loss of supporting alveolar bone in some end-stage periodontitis. The aim of this study is to report a kind of treatment modality which was seemed to be successes full in maintaining teeth. In this case-report, a hopeless tooth was saved by combined non-surgical periodontalendodontal treatment and antimicrobial photodynamic therapy (aPDT).

Case Report: A 58-year-old male presented with a chief complaint of pain and mobility of tooth number 38. Clinical examinations revealed a periodontic-endodontic lesion with clinical attachment loss exceeding $10 \mathrm{~mm}$ and grade III mobility. To preserve the tooth, we operated nonsurgical periodontal treatment including scaling and root planning (SRP) plus root canal therapy (RCT) combined with intra-root canal non-aPDT laser decontamination. Then we applied laser pocket therapy with and without aPDT. Following 6 months of the aPDT treatment, the mobility and pocket depth of the tooth improved from grade III to I and from 10 to 3 millimeters respectively. Conclusion: aPDT is a novel adjunctive therapy that can be used for various conditions with microbial etiology. This case report demonstrated that aPDT might be effective in the treatment of periodontic-endodontic lesions in a hopeless tooth.

Keywords: Antimicrobial photodynamic therapy, Diode laser, Periodontic-endodontic lesion, Furcation involvement, Periodontitis
\end{abstract}

\section{Introduction}

Periodontitis is an inflammatory disease that involves the supporting tissues of the tooth, leading to the progressive destruction of the underlying periodontal apparatus and consequently attachment loss. In spite of some ambiguity in the definition, classification and prevalence of periodontitis, it is a major world health issue. According to the World Health Organization Global Data Bank, the prevalence of advanced periodontitis ranges from 1\%$79 \%$ worldwide. ${ }^{1}$ If periodontitis is left untreated, it could spread inflammation and infection throughout the body and lead to pain, discomfort, tooth loose, difficulty in eating, esthetic problems, and ultimately reduced quality of life. ${ }^{2}$

The current gold standard treatment of periodontitis is the non-surgical removal of supra and sub-gingival periodontal biofilms by scaling and root planning (SRP) with both of hand and ultrasonic instruments ${ }^{3}$. SRP disrupts microbial biofilms, increases the clinical attachment level (CAL), and leads to the recession of gingival margin with an inflammation decline. ${ }^{4}$ In some conditions such as resistant periodontitis, deep periodontal pockets or complex root tooth anatomy, SRP could not efficiently eliminate microbial biofilms. ${ }^{5}$ In most cases, the pocket depths of $7 \mathrm{~mm}$ or more are not accessible for mechanical debridement without periodontal flap surgery. Therefore, surgical methods are mandatory to reach predictable results in such deep periodontal pockets. Surgical approaches are reserved for the last line of periodontal treatment because they are aggressive and have many complications leading to discomfort and the high cost of treatment. Thus, finding conservative adjunctive treatments are valuable steps toward raising the scope of periodontal therapies. ${ }^{6}$

To date, various adjuncts have been emerged to improve SRP treatment gaps, such as systemic and local antiinfective (AIN) chemotherapeutic agents, the nonsurgical application of lasers (particularly diode lasers for both 
aPDT and Non-aPDT), and treatment modalities based on host modulation, including Bisphosphonates and Non-steroidal anti-inflammatory drugs. ${ }^{7}$

Systemic AIN chemotherapy with antibiotic (ABs) drugs is one of the most effective adjunctive available methods. The penetrative potential of systemic ABs makes it a powerful adjunct to the SRP treatment. ${ }^{7}$ Adjunctive systemic or local AIN chemotherapy significantly improves the mean periodontal pocket depth (PPD), CAL, and bleeding on probing. ${ }^{4,8-10}$ Depends on different types of systemic ABs, the mean CAL improvement are ranged from 0.09 to $1.10 \mathrm{~mm}^{11}$ and for adjunctive local $\mathrm{AB}, \mathrm{CAL}$ gain are ranged from 0.5 to $1 \mathrm{~mm} .{ }^{10,12}$

Another novel therapeutic approach for nonsurgical periodontal treatment is phototherapy by laser technology. There are little pieces of evidence supporting the use of the laser alone (non-aPDT) as an adjunct to the SRP treatment. ${ }^{13}$

After ABs, photodynamic therapy (PDT) or photochemotherapy has emerged to overcome the AIN chemotherapy and Laser alone limitations. PDT was introduced for the first time as a non-invasive localized therapeutic modality for cancer treatment in the early 1900s. ${ }^{14}$ Later, its local chemotherapeutic and antimicrobial effect was utilized. It operates by the interaction of three elements: a photosensitizer (PS), reactive oxygen species (ROS) and a low-intensity or infra-red laser beam. PS is taken up by peculiar microorganisms and is activated by a laser beam with a specific wavelength to the particular PS. When the PS is activated, it produces ROS which is toxic to microorganisms. ${ }^{15,16}$

The application of aPDT in chronic periodontitis is based on the proved sensitivity of some of the microbial pathogens to aPDT in in vitro and animal studies. Some of these microorganisms such as Aggregatibacter actinomycetemcomitans, Porphyromonas gingivalis, and Fusobacterium nucleatum have the fundamental etiopathogenic role in chronic periodontitis. ${ }^{17,18}$ Therefore, aPDT acts as a targeted therapy in periodontics, a specific feature that none of the antibiotics have it.

Some advantages of aPDT to AIN therapy are efficacy against resistant bacteria, safety to the surrounding tissues, absence of systemic side effects, rapid bactericidal effect during periodontal procedures and no need for local anesthesia. ${ }^{9,19}$

Similar to periodontitis, microbial infections play an important role in persistent perapical lesions with pulpal origin. ${ }^{20}$ Endodontic treatments are not with a high success rate (about $40-80 \%$ ), particularly in the Iranian population (with $52 \%$ failure). ${ }^{21-25}$ Factors such as the formation of bacterial biofilm, especially in dentinal tubules, the existence of anatomical complexities, and resistant bacteria such as Enterococcus faecalis are related to root canal therapy (RCT) failures. ${ }^{26,27}$ Various chemical and anti-infective materials have been used to treat such refractory cases. ${ }^{28,29}$ In recent years, PDT has been introduced as an adjunct in endodontic treatment with acceptable success ${ }^{25,30}$ so that PDT was reported to be effective in eliminating Enterococcus faecalis from infected root canals in several studies. ${ }^{31}$

The current issue presents a case of periodonticendodontic lesion of a hopeless tooth which was treated by SRP with the adjuncts of aPDT and Non-aPDT laser pocket therapy and intra-root canal laser decontamination.

\section{Case Report}

A 58-year-old male was presented to our private clinic in July 2017 with a chief complaint of mobility and pain of tooth number 38 . His past medical history was unremarkable and he had not a-history of smoking or drinking. Intraoral examination was in normal limits, except for grade III mobility of tooth number 38, grade III furcation involvement, periodontal pockets and attachment losses exceeding $10 \mathrm{~mm}$ in several tooth line angles.

Radiographic evaluation by panoramic modality showed moderate resorption of alveolar bone in all molar regions, especially in the left upper and lower quadrants. Periapical radiography of tooth number 38 showed a welldemarcated and non-corticated radiolucency surrounding both roots and its furcation from crestal alveolar bone to the periapical areas (vertical bone loss). Surrounding anatomic structures were not affected; therefore, any findings of cortical bone expansion, root resorption, and inferior alveolar canal or tooth displacement were not evident (Figure 1).

The radiolucent lesion seemed to have inflammatory nature due to the negative results of third molar vitality tests. Therefore, based on dental history, clinical tests and the radiographs of tooth 38 , the diagnosis was a periodontic-endodontic lesion of type 3 , according to Grossman classification. ${ }^{32}$ Consequently, the tooth had a hopeless prognosis, which had not adequate attachment to maintain function, health and comfort and had to be extracted.

Some clues such as bilateral alveolar bone losses and muscular face appearance with hypertrophic masseter muscles fortify trauma from occlusion as an etiology of the diagnosis. Despite suggesting dental implant as the

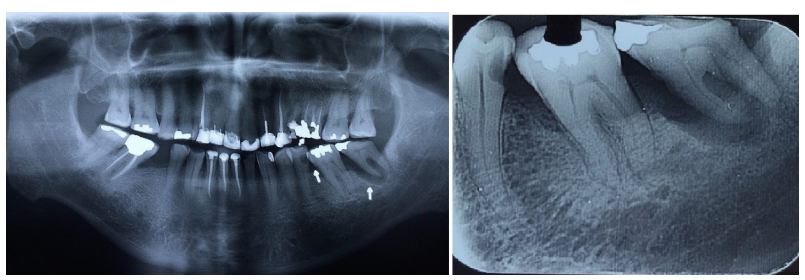

Figure 1. (a) The panoramic view that shows the moderate resorption of alveolar bone at molar teeth regions, especially in the left upper and lower quadrants. (b) The periapical radiography of tooth number 38 shows a welldemarcated and non-corticated radiolucency surrounding both roots and its furcation from crestal alveolar bone to the Periapical areas. 
first choice of treatment, the patient disagreed and his willingness to save the tooth encouraged us to precede non-surgical treatment. In this case, the third molar had been migrated to the anatomic site of the previously extracted second molar; thus saving the third molar was beneficial to restoring similar functions.

Before any intervention, the patient signed informed consent, and this study followed the principles of the revised Helsinki Declaration in 2013. ${ }^{33}$ Any surgical measures attempting to save the third molar could lead to peeling the tooth off. Therefore, we decided to perform non-surgical and conservative periodontal treatment along with RCT by means of the diode laser as an adjunctive treatment. Accordingly, phase I periodontal therapy began by the elimination of the trauma as the main etiologic factor for periodontal involvement with occlusal adjustment.

Biomechanical decontamination of the periodontal pockets was done by scaling and root debridement combined with non-aPDT (pocket laser therapy) with $980 \mathrm{~nm}$ diode laser beam irradiation. The diode laser parameters (Wiser, DoctorSmile, Italy) were as follows: $2.5 \mathrm{~W}$ of power with $0.7 \mathrm{~W}$ average, time on (Ton) of 30 seconds and time off (Toff) of 70 seconds (Figure 2) and the optic fiber movement speed was $2 \mathrm{~mm} / \mathrm{s}$.

After some improvement of tooth mobility, RCT performed and the $980 \mathrm{~nm}$ diode laser was used for intra-root canal disinfection and conditioning. In this procedure, the power density of the diode laser was adjusted to $2.5 \mathrm{~W}$ in a continuous mode. Special endodontic fiber with $200 \mathrm{~mm}$ width was recruited for intra-root canal irradiation. It moved from the near apical root area to the coronal region, with spiral motion and the movement speed of $2 \mathrm{~mm} / \mathrm{s}$.

After 2 weeks, adjunctive aPDT was employed by the diode laser. Methylene blue (Blue $+\mathrm{M}$, NovaTebPars, Iran) at concentration of $(100 \mu \mathrm{g} / \mathrm{mL})$ was-utilized as a PS substance and injected into the periodontal pocket. The laser tip movement was 360 degrees around the whole area of the pocket. The diode laser of a $660 \mathrm{~nm}$ wavelength was emitted in each pocket for 60 seconds. Power and energy were $150 \mathrm{~mW}$ and $9 \mathrm{~J}$ respectively (Figure 3 ).

Preventing eye damage, the patient and clinicians wore
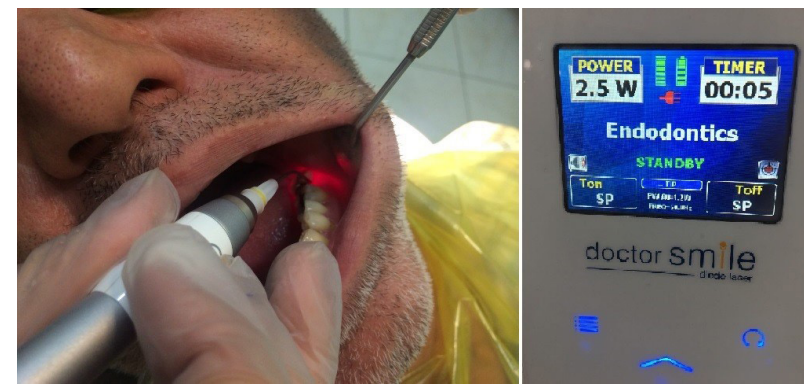

Figure 2. The power of the diode laser for Non-aPDT for pocket therapy was $2.5 \mathrm{~W}$ and the average power was $(\mathrm{AV})=0.7 \mathrm{~W}$. specific protecting eyeglasses during all of such laser applications. The patient was informed about the hopeless condition of the tooth and all of the procedures were explained and informed consent was taken. Following 6 months of therapy, tooth mobility was reduced from grade III to I and the-periodontal pocket decreased from $10 \mathrm{~mm}$ to less than $3 \mathrm{~mm}$ (Figure 4). Pain and discomfort were resolved after RCT. Radiographic evaluation after 6 months of therapy showed significant shrinkage of the periodontic-endodontic lesion with vertical and horizontal alveolar bone regeneration (Figure 5). These promising results even with periodontal open flap surgery were not accessible.

\section{Discussion}

In periodontitis, the majority of studies have demonstrated the significant beneficial effect of aPDT as an adjunct to the SRP in favor of lowering PD and increasing CAL during a 3 to 6-month follow-up. ${ }^{5,14,16,34}$ Based on a recent systematic review, most of the supportive measures, including aPDT, could improve SRP treatment results in terms of CAL improvement from 0.2 to $0.6 \mathrm{~mm}$ over SRP alone. ${ }^{34}$ A recent systematic review and metaanalysis compared the adjunctive role of antibiotics

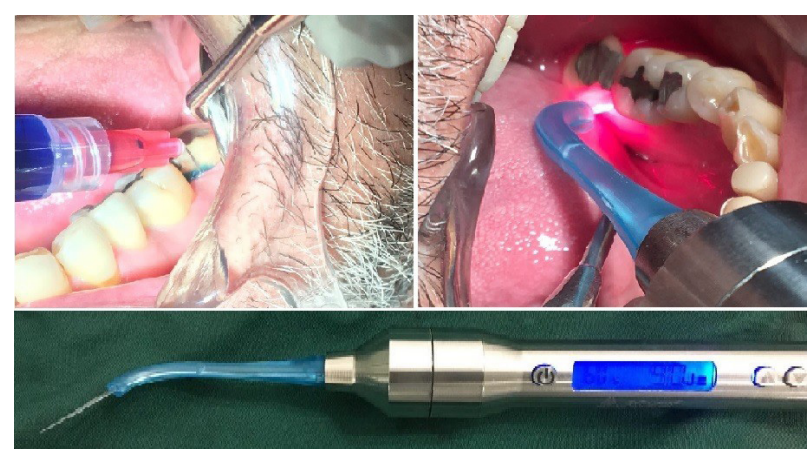

Figure 3. aPDT was employed by a diode laser with the following settings (a): Methylene blue $(100 \mu \mathrm{g} / \mathrm{mL})$ was used as a photosensitizer substance and injected into the periodontal pocket. (b): The diode laser was utilized with $660 \mathrm{~nm}$ wavelengths, 60 seconds duration per each pocket, power of $150 \mathrm{~mW}$, and energy of $9 \mathrm{~J}$. (c): The laser tip movement was 360 degrees around the whole area of the tooth pocket.
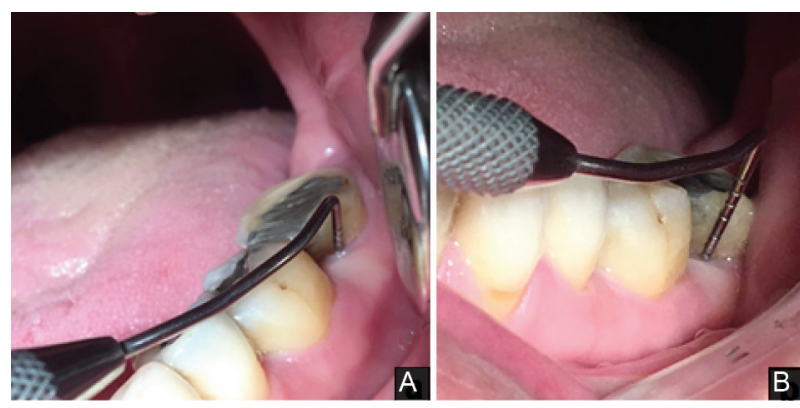

Figure 4. Decreasing the Probing Depth of the Right 3rd Molar Following Antimicrobial Photodynamic Therapy. (A) Shows before and (B) shows after the treatment of the probing depth. 


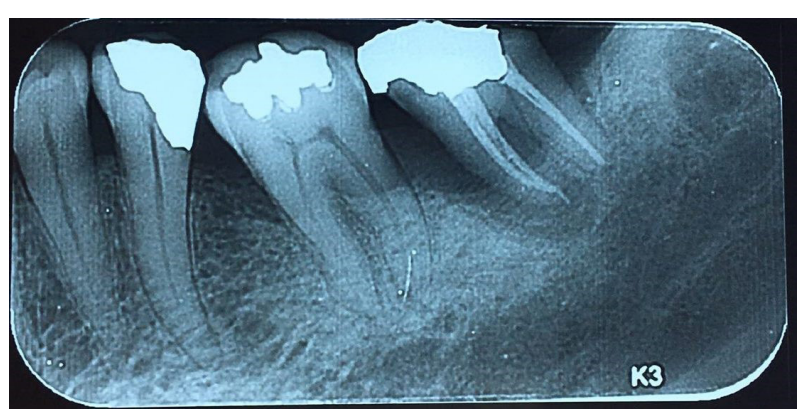

Figure 5. The periapical radiography of tooth number 38 following six months of treatment showed significant shrinkage of the periodonticendodontic lesion with vertical and horizontal alveolar bone formation.

versus aPDT to the SRP treatment. In this research, none of the five included studies showed extra advantages of aPDT at the follow-up. A meta-analysis comparing the effect of $\mathrm{AB}$ and $\mathrm{aPDT}$ on $\mathrm{PPD}$ respectively showed a high and a low degree of heterogeneity on CAL in both groups. Comparing the two groups, PPD was not reduced significantly at the follow-up, but CAL increased significantly when adjunctive aPDT was used. ${ }^{9}$

Altogether lack of enough clinical trials with head to head comparison methods and a high degree of heterogeneity between studies make the comparison of several adjunctive methods to the SRP treatment difficult. Nowadays for choosing the best approach, a specialist needs to consider many clinical, radiographic data and review the systems to plan the most effective treatment.

In our study, we used non-aPDT and aPDT treatment for periodontal pocket therapy and non-aPDT for endodontic disinfection simultaneously in a tooth with periodontic-endodontic lesion and furcation involvement. To our knowledge, this method of using three approaches together is novel and has not been used before.

Very few studies are available about the effect of aPDT on furcation involvement. In a clinical trial by Andrade and colleagues, they found that aPDT can modulate gene expression during the periodontal treatment of class III furcation involvement, which inhibits connective tissue and alveolar bone destruction. ${ }^{35}$ Another double-blind, parallel, randomized controlled clinical trial by Luchesi and colleagues showed that aPDT could not promote classic SRP treatment benefits for class II furcation, but it presented a reduction in periodontal pathogens and a gain in the local levels of cytokines. ${ }^{36}$

In a multicenter study, systemic $A B$ therapy with amoxicillin plus metronidazole could not improve the furcation involvement significantly in comparison with the placebo. ${ }^{37}$

In our study, CAL gain was $7 \mathrm{~mm}$ within a 3 -month follow-up and these promising results in CAL and tooth mobility improvement have not been reported in the literature yet.

In spite of the heterogeneity in different studies and the absence of standard and widely accepted guidelines for aPDT in chronic periodontitis treatment, we followed the most accepted methods in systematic reviews and meta-analysis studies regarding the laser parameters such as laser wavelength, duration and frequency of laser application, laser fiber tip diameter, power density, type of PS (methylene blue) and its concentration. . $^{14,16}$

We applied a $660 \mathrm{~nm}$ wavelength diode laser for aPDT. This wavelength is well transmitted through the tissues to activate the absorbed PS. ${ }^{19}$

A $980 \mathrm{~nm}$ diode laser has several benefits for the decontamination of the intra-periodontal pocket and intra-root canal tooth application. A $980 \mathrm{~nm}$ diode laser can penetrate 2-3 $\mathrm{mm}$ depth through epithelial and subepithelial tissues and could be absorbed by soft tissue rather than hard tissue, which makes it suitable for disinfecting properties. ${ }^{38}$

To our knowledge, there is no report of using aPDT in the teeth with periodontic-endodontic or endodonticperiodontic lesions or with grade III mobility, furcation involvement and a hopeless prognosis in the literature. This makes our case report unique in that using aPDT for preserving a hopeless tooth.

Although we could not generalize our work results to other teeth with such hopeless conditions, this case report could lead future researches to employ aPDT as a new regenerative treatment and an evolutionary technique for preserving teeth instead of the dental implant as an aggressive and expensive treatment.

\section{Ethical Considerations}

Written consent was obtained from the patient prior to any treatment procedures.

\section{Conflict of Interests}

None declared.

\section{Acknowledgments}

The authors gratefully acknowledge the patient for his participation in this study.

\section{References}

1. Periodontal country profiles: an overview of CPITN data in the WHO Global OralData Bank [Internet]. 2016. Available from: https://www5.dent.niigata-u.ac.jp/ prevent/perio/ contents.html. Accessed 25 July 2016.

2. Manresa C, Sanz-Miralles EC, Twigg J, Bravo M. Supportive periodontal therapy (SPT) for maintaining the dentition in adults treated for periodontitis. Cochrane Database Syst Rev. 2018;2018(1):CD009376. doi: 10.1002/14651858. CD009376.pub2.

3. Atieh MA. Photodynamic therapy as an adjunctive treatment for chronic periodontitis: a meta-analysis. Lasers Med Sci. 2010;25(4):605-13. doi: 10.1007/s10103009-0744-6.

4. Keestra JA, Grosjean I, Coucke W, Quirynen M, Teughels W. Non-surgical periodontal therapy with systemic 
antibiotics in patients with untreated chronic periodontitis: a systematic review and meta-analysis. J Periodontal Res. 2015;50(3):294-314. doi: 10.1111/jre.12221.

5. Azaripour A, Dittrich S, Van Noorden CJF, Willershausen B. Efficacy of photodynamic therapy as adjunct treatment of chronic periodontitis: a systematic review and metaanalysis. Lasers Med Sci. 2018;33(2):407-23. doi: 10.1007/ s10103-017-2383-7.

6. O'Rourke VJ. Azithromycin as an adjunct to non-surgical periodontal therapy: a systematic review. Aust Dent J. 2017;62(1):14-22. doi: 10.1111/adj.12448.

7. Newman MG, Takei HH, Klokkevold PR, Carranza FA. Newman and Carranza's clinical periodontology. 13th ed. St. Louis, Missouri: Elsevie; 2019.

8. Rovai ES, Souto ML, Ganhito JA, Holzhausen M, Chambrone L, Pannuti CM. Efficacy of local antimicrobials in the non-surgical treatment of patients with periodontitis and diabetes: A systematic review. J Periodontol. 2016;87(12):1406-17. doi: 10.1902/jop.2016.160214.

9. Akram Z, Hyder T, Al-Hamoudi N, Binshabaib MS, Alharthi SS, Hanif A. Efficacy of photodynamic therapy versus antibiotics as an adjunct to scaling and root planing in the treatment of periodontitis: a systematic review and meta-analysis. Photodiagnosis Photodyn Ther. 2017;19:8692. doi: 10.1016/j.pdpdt.2017.05.007.

10. Chambrone L, Vargas M, Arboleda S, Serna M, Guerrero M, de Sousa J, et al. Efficacy of local and systemic antimicrobials in the non-Surgical treatment of smokers with chronic periodontitis: a systematic review. J Periodontol. 2016;87(11):1320-32. doi: 10.1902/ jop.2016.160268.

11. Haffajee AD, Socransky SS, Gunsolley JC. Systemic anti-infective periodontal therapy. A systematic review. Ann Periodontol. 2003;8(1):115-81. doi: 10.1902/ annals.2003.8.1.115.

12. Smiley CJ, Tracy SL, Abt E, Michalowicz BS, John MT, Gunsolley J, et al. Systematic review and meta-analysis on the nonsurgical treatment of chronic periodontitis by means of scaling and root planing with or without adjuncts. J Am Dent Assoc. 2015;146(7):508-24 e5. doi: 10.1016/j. adaj.2015.01.028.

13. Cobb CM, Low SB, Coluzzi DJ. Lasers and the treatment of chronic periodontitis. Dent Clin North Am. 2010;54(1):3553. doi: 10.1016/j.cden.2009.08.007.

14. Xue D, Tang L, Bai Y, Ding Q, Wang P, Zhao Y. Clinical efficacy of photodynamic therapy adjunctive to scaling and root planing in the treatment of chronic periodontitis: A systematic review and meta-analysis. Photodiagnosis Photodyn Ther. 2017;18:119-27. doi: 10.1016/j. pdpdt.2017.01.183.

15. Freitas PM, Simões A, eds. Lasers in Dentistry: Guide for Clinical Practice. Ames, Iowa: Wiley-Blackwell; 2015. doi: 10.1002/9781118987742.

16. Sgolastra F, Petrucci A, Severino M, Graziani F, Gatto $\mathrm{R}$, Monaco A. Adjunctive photodynamic therapy to non-surgical treatment of chronic periodontitis: a systematic review and meta-analysis. J Clin Periodontol. 2013;40(5):514-26. doi: 10.1111/jcpe.12094.

17. Sigusch BW, Engelbrecht M, Völpel A, Holletschke A, Pfister W, Schütze J. Full-mouth antimicrobial photodynamic therapy in Fusobacterium nucleatum-infected periodontitis patients. J Periodontol. 2010;81(7):975-81. doi: 10.1902/ jop.2010.090246.

18. Theodoro LH, Silva SP, Pires JR, Soares GH, Pontes AE, Zuza EP, et al. Clinical and microbiological effects of photodynamic therapy associated with nonsurgical periodontal treatment. A 6-month follow-up. Lasers Med Sci. 2012;27(4):687-93. doi: 10.1007/s10103-011-0942-x.

19. Raghavendra M, Koregol A, Bhola S. Photodynamic therapy: a targeted therapy in periodontics. Aust Dent J. 2009;54(Suppl 1):S102-9. doi: 10.1111/j.18347819.2009.01148.x.

20. Siqueira JF Jr. Endodontic infections: concepts, paradigms, and perspectives. Oral Surg Oral Med Oral Pathol Oral Radiol Endod. 2002;94(3):281-93. doi: 10.1067/ moe.2002.126163.

21. Boucher Y, Matossian L, Rilliard F, Machtou P. Radiographic evaluation of the prevalence and technical quality of root canal treatment in a French subpopulation. Int Endod J. 2002;35(3):229-38. doi: 10.1046/j.1365-2591.2002.00469.x.

22. Kirkevang LL, Hörsted-Bindslev P, Ørstavik D, Wenzel A. A comparison of the quality of root canal treatment in two Danish subpopulations examined 1974-75 and 199798. Int Endod J. 2001;34(8):607-12. doi: 10.1046/j.13652591.2001.00436.x.

23. Dugas NN, Lawrence HP, Teplitsky PE, Pharoah MJ, Friedman S. Periapical health and treatment quality assessment of root-filled teeth in two Canadian populations. Int Endod J. 2003;36(3):181-92. doi: 10.1046/j.13652591.2003.00640.x.

24. Asgary S, Shadman B, Ghalamkarpour Z, Shahravan A, Ghoddusi J, Bagherpour A, et al. Periapical status and quality of root canal fillings and coronal restorations in iranian population. Iran Endod J. 2010;5(2):74-82.

25. Asnaashari M, Mojahedi SM, Asadi Z, Azari-Marhabi S, Maleki A. A comparison of the antibacterial activity of the two methods of photodynamic therapy (using diode laser $810 \mathrm{~nm}$ and LED lamp $630 \mathrm{~nm}$ ) against Enterococcus faecalis in extracted human anterior teeth. Photodiagnosis Photodyn Ther. 2016;13:233-7. doi: 10.1016/j. pdpdt.2015.07.171.

26. Hancock HH 3rd, Sigurdsson A, Trope M, Moiseiwitsch J. Bacteria isolated after unsuccessful endodontic treatment in a North American population. Oral Surg Oral Med Oral Pathol Oral Radiol Endod. 2001;91(5):579-86. doi: 10.1067/ moe.2001.113587.

27. Siqueira JF Jr, Araújo MC, Garcia PF, Fraga RC, Dantas CJ. Histological evaluation of the effectiveness of five instrumentation techniques for cleaning the apical third of root canals. J Endod. 1997;23(8):499-502. doi: 10.1016/ s0099-2399(97)80309-3.

28. Byström A, Sundqvist G. Bacteriologic evaluation of the effect of 0.5 percent sodium hypochlorite in endodontic therapy. Oral Surg Oral Med Oral Pathol. 1983;55(3):30712. doi: 10.1016/0030-4220(83)90333-x.

29. Neelakantan P, Romero M, Vera J, Daood U, Khan AU, Yan A, et al. Biofilms in endodontics-current status and future directions. Int J Mol Sci. 2017;18(8):1748. doi: 10.3390/ ijms18081748.

30. Asnaashari M, Godiny M, Azari-Marhabi S, Tabatabaei FS, Barati M. Comparison of the antibacterial effect of 810 $\mathrm{nm}$ diode laser and photodynamic therapy in reducing the 
microbial flora of root canal in endodontic retreatment in patients with periradicular lesions. J Lasers Med Sci. 2016;7(2):99-104. doi: 10.15171/jlms.2016.17.

31. Siddiqui SH, Awan KH, Javed F. Bactericidal efficacy of photodynamic therapy against Enterococcus faecalis in infected root canals: a systematic literature review. Photodiagnosis Photodyn Ther. 2013;10(4):632-43. doi: 10.1016/j.pdpdt.2013.07.006.

32. Tsesis I, Nemcovsky CE, Nissan J, Rosen E. EndodonticPeriodontal Lesions: Evidence-Based Multidisciplinary Clinical Management. 2019

33. World Medical Association Declaration of Helsinki: ethical principles for medical research involving human subjects. JAMA. 2013;310(20):2191-4.

34. Smiley CJ, Tracy SL, Abt E, Michalowicz BS, John MT, Gunsolley J, et al. Evidence-based clinical practice guideline on the nonsurgical treatment of chronic periodontitis by means of scaling and root planing with or without adjuncts. J Am Dent Assoc. 2015;146(7):525-35. doi: 10.1016/j. adaj.2015.01.026.
35. Andrade PF, Garlet GP, Silva JS, Fernandes PG, Milanezi $\mathrm{C}$, Novaes AB Jr, et al. Adjunct effect of the antimicrobial photodynamic therapy to an association of non-surgical and surgical periodontal treatment in modulation of gene expression: a human study. J Photochem Photobiol B. 2013;126:119-25. doi: 10.1016/j.jphotobiol.2013.06.012.

36. Luchesi VH, Pimentel SP, Kolbe MF, Ribeiro FV, Casarin RC, Nociti FH Jr, et al. Photodynamic therapy in the treatment of class II furcation: a randomized controlled clinical trial. J Clin Periodontol. 2013;40(8):781-8. doi: 10.1111/jcpe.12121.

37. Eickholz P, Nickles K, Koch R, Harks I, Hoffmann $\mathrm{T}$, Kim TS, et al. Is furcation involvement affected by adjunctive systemic amoxicillin plus metronidazole? A clinical trials exploratory subanalysis. J Clin Periodontol. 2016;43(10):839-48. doi: 10.1111/jcpe.12594.

38. Derikvand N, Ghasemi SS, Moharami M, Shafiei E, Chiniforush N. Management of oral lichen planus by 980 nm diode laser. J Lasers Med Sci. 2017;8(3):150-4. doi: 10.15171/jlms.2017.27. 